\title{
Sorting of spatially incoherent optical vortex modes
}

\author{
Daniel O. Bezerra ${ }^{1}$, Joao P. Amaral ${ }^{1}$, Eduardo J. S. Fonseca ${ }^{1}$, Cleberson R. Alves ${ }^{2} \&$ \\ Alcenísio J. Jesus-Silva ${ }^{1 *}$
}

Coherent optical vortices have promising applications in quantum and classical optical communication. They add new degrees of freedom to code information. In this context, to implement a tool enabling sorting of spatially multiplexed vortex states is fundamental. By other hand, spatially incoherent vortices can be more robust in propagation through noise media, such as turbulent atmosphere or obstacles that block part of the light. Therefore, in this work we propose directly applying a highresolution sorting scheme to spatially incoherent vortex states.

\begin{abstract}
Since the discovery of the orbital angular momentum (OAM) of light or optical vortices ${ }^{1}$, one application that have attracted more attention recently is its use as a new degree of freedom to burst optical communications in the classical ${ }^{2,3}$ and quantum regimes ${ }^{4,5}$. Several orthogonal modes can be exploited to spatial multiplexing and increase the transmission capacity in optical communication systems. However, the most used optical beam is the OAM beam called Laguerre-Gauss (LG) beam ${ }^{6}$ which can be decomposed in terms of orthogonal components allowing being space-divided with low inter-modal crosstalk among multiple modes ${ }^{2,3,7}$. This beam has a finite spatial extend and due to its azimuthal phase dependence can be sorted ${ }^{8-10}$, being ideal to optical communications.

There are several proposals for OAM sorting. For example, the interferometry methods ${ }^{11,12}$, which require increasing complexity of the scheme with increasing number of modes to be sorted. However, the best approach to OAM sorting is based on a ray-optics coordinate transformation. To date, there are two types of such mode sorting. The first is based on log-polar transformation ${ }^{13}$ which can map OAM modes with an azimuthal phase to plane waves with tilts proportional to the vortex topological charge (TC) ${ }^{8}$. This approach is simple and efficient, but there is a limited separation of adjacent modes. One work improved this result at the cost of adding an additional phase-only hologram known as fan-out element that makes copies of the input optical fields and the interference of these copies can produce a better resolution ${ }^{9}$, but it also increase the complexity of the scheme. The second type of ray-optics coordinate transformation is the spiral transformation that maps spirals to parallel lines. In this case, the tilt of the plane waves is theoretically unlimited and, in practice, the resolution of the mode separation is considerably improved ${ }^{10}$.

Spatially incoherent optical vortices, are vortices originated by passing an incoherent light through a hologram designed to generate optical vortices ${ }^{14}$ or when a coherent optical vortex is scattered by a rough surface $e^{15-18}$. Those works have shown that the generated light presents a vortex in the coherence function, the so-called coherence vorte $^{19}$. Remarkable, the lower coherence vortices are less influenced than those of higher coherence during propagation through turbulent atmosphere ${ }^{20}$ and through obstacles ${ }^{21}$. Therefore, discriminating incoherent optical vortices with different superimposed TCs is a topic of current interest for optical communication through turbulent atmosphere ${ }^{22,23}$.
\end{abstract}

\section{Results}

Concept and principle. The spiral transformation method has been successfully applied for high resolution sorting of coherent OAM modes of light ${ }^{10}$. In this work we have applied it to OAM mode sorting of spatially incoherent OAM modes. Figure 1 illustrates the main idea of the present work. A spatially incoherent light, which actually is a speckle field, is used with a hologram designed to generate LG beams. Therefore, incoherent LG beams are generated and their optical Fourier transform ${ }^{24}$ is projected over the transformation phase mask $Q(x, y)$. The resulting field has its optical Fourier transform projected over the correction phase mask $P(x, y)$. A final optical Fourier transform generates a final speckle field. The intensity of the final speckle field fluctuates 


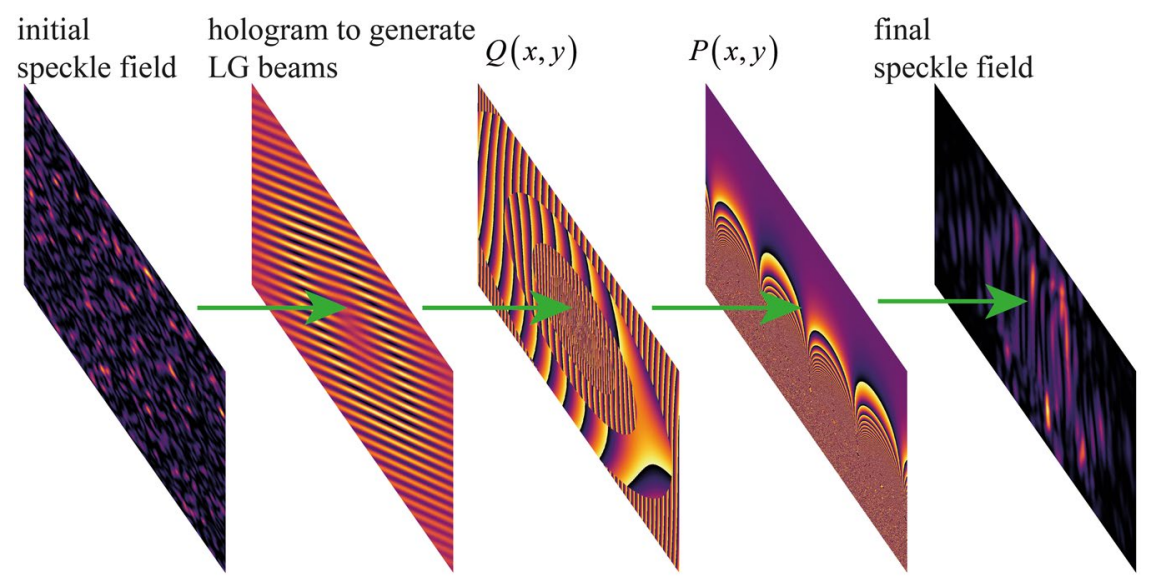

Figure 1. The general idea of the proposal: a spatially incoherent light represented by a speckle field, instead of coherent light, is used to generate OAM modes. The incoherent modes are passed through a usual system of coherent mode sorting and a final speckle field is produced from which we have to extract the information of the sorted modes.

randomly, but it still has the information of the sorted modes. This information is recovered from the correlations of the spatial intensity fluctuations ${ }^{25}$.

The spiral transformation is generated by a phase mask, which is described by the following equation ${ }^{10}$,

$$
Q(x, y)=\frac{k \beta}{f\left(a^{2}+1\right)}\left[(a x+y) \ln \left(\frac{r}{r_{0}}\right)+(x-a y) \theta-(a x+y)\right],
$$

where $k=2 \pi / \lambda$ and $\lambda$ is the light wavelength, $f$ is the focal length of a lens put at a distance $f$ behind the phase element to perform its Fourier transform ${ }^{24}$, and $a, \beta$ and $r_{0}$ are constants. The $r$ and $\theta$ are the polar coordinates but the variable $\theta$ is not the conventional one since it is unlimited,

$$
r=\left(x^{2}+y^{2}\right)^{1 / 2}, \theta=\theta_{0}+2 m \pi,
$$

where

$$
\theta_{0}=\tan ^{-1}\left(\frac{y}{x}\right) \in[0,2 \pi), m=\left\lfloor\frac{1}{2 \pi a} \ln \left(\frac{r}{r_{0}} e^{-a \theta_{0}}\right)\right\rfloor
$$

and $\rfloor$ is the floor function. A second phase element is used as a phase-correction mask such that its local gradient at a point $(u ; v)$ compensate the slope of the ray arriving at this point from the corresponding point $(x ; y)$ in the previous phase element ${ }^{10}$. This phase-correction element is given by,

$$
P(u, v)=\frac{k r_{0}}{f} \frac{\beta}{1+a^{2}} \exp \left(\frac{a u+v}{\beta}\right)\left[\sin \left(\frac{u-a v}{\beta}\right)+a \cos \left(\frac{u-a v}{\beta}\right)\right]
$$

where the constants are the same defined for the $Q(x, y)$ function and $(u, v)$ are the spatial coordinates at the plane of the phase-correction element.

Experimental setup. The Fig. 2 displays the used experimental setup. A laser beam is expanded and collimated by a telescope formed through lenses specified by its focal length $f_{1}=2.8 \mathrm{~mm}$ and $f_{2}=150 \mathrm{~mm}$, where a rotating ground glass disc (RGGD) is placed between these lenses near the focus of the lens of focal length $f_{1}$. In order to get the best mode distinguishability we make use of the control of the source size ${ }^{26,27}$ slightly dislocating the RGGD from the focus. The obtained light illuminates the right half of $S L M_{1}$ which was half divided in order to contain, in the right, the hologram to generate LG beams ${ }^{28}$ and, in the left, the $Q(x, y)$ phase. The generated LG beam is Fourier transformed and projected over $Q(x, y)$ by a lens of focal length $f_{4}=1000 \mathrm{~mm}$.

After the second time reflecting in the $S L M_{1}$ the LG beams acquire a phase $Q(x, y)$ and a lens of focal length $f_{3}=300 \mathrm{~mm}$ projects the Fourier transform of this new field over the $S L M_{2}$ which contain the correction phase $P(u, v)$. A lens of focal length $f_{5}=200 \mathrm{~mm}$ projects the Fourier transform of the field reflected from the $S L M_{2}$ on the CCD camera. To calculate the phase elements $Q(x, y)$ and $P(u, v)$ we have used $f=f_{5}=200 \mathrm{~mm}$, $a=\ln (1.6) /(2 \pi), \beta=1.8 /(2 \pi) m m$ and $r_{0}=1.1 \mathrm{~mm}$. 


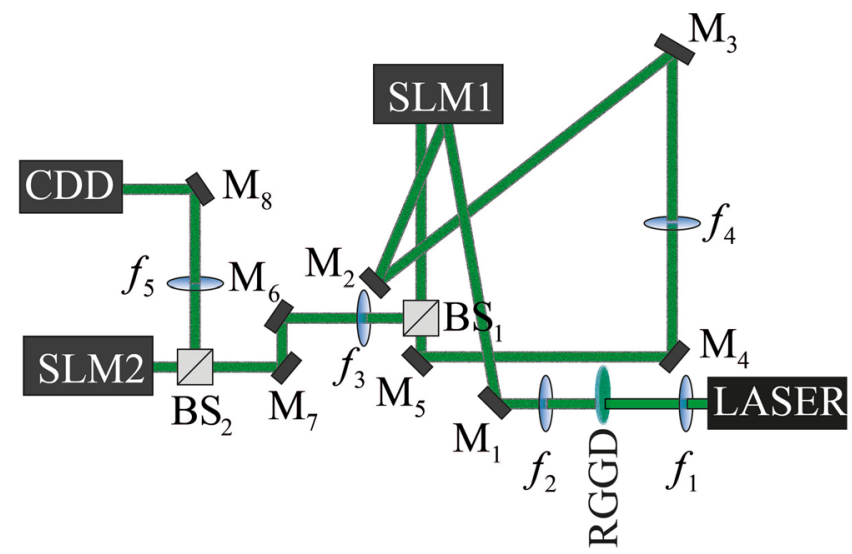

Figure 2. Experimental setup: $S L M_{1}$ and $S L M_{2}$ are spatial light modulators, $C C D$ stands for charge coupled device camera, $M_{1}$ to $M_{8}$ are mirrors, $B S_{1}$ and $B S_{2}$ are beam splitters, the focal length of the lenses are $f_{1}=2.8 \mathrm{~mm}, f_{2}=150 \mathrm{~mm}, f_{3}=300 \mathrm{~mm}, f_{4}=1000 \mathrm{~mm}, f_{5}=200 \mathrm{~mm}$.

Theory. We present the theoretical formulation of the conceptual idea of the experiment with spatially incoherent light. The speckle field $E_{1}\left(\mathbf{r}_{1}\right)$ generated in the RGGG hits the first half of the SLM1 containing a hologram that encode the signal field $S\left(\mathbf{r}_{1}\right)$, i.e., LG modes. The resulting field is Fourier transformed by a lens of focal length $f_{4}$,

$$
E_{2}\left(\mathbf{r}_{2}\right)=\int E_{1}\left(\mathbf{r}_{1}\right) S\left(\mathbf{r}_{1}\right) \exp \left(\frac{2 \pi i}{\lambda f_{4}} \mathbf{r}_{1} \cdot \mathbf{r}_{2}\right) d \mathbf{r}_{1} .
$$

The field $E_{2}\left(\mathbf{r}_{2}\right)$ is projected in the second half of the SLM1 containing the phase $Q\left(\mathbf{r}_{2}\right)$. The resulting field is Fourier transformed by a lens of focal length $f_{3^{\prime}}$,

$$
E_{3}\left(\mathbf{r}_{3}\right)=\int E_{2}\left(\mathbf{r}_{2}\right) \exp \left[i Q\left(\mathbf{r}_{2}\right)\right] \exp \left(\frac{2 \pi i}{\lambda f_{3}} \mathbf{r}_{2} \cdot \mathbf{r}_{3}\right) d \mathbf{r}_{2} .
$$

The field in Eq. (6) hits the SLM2 which contains the phase $P\left(\mathbf{r}_{3}\right)$. The resulting field is Fourier transformed by a lens of focal length $f_{5}$ producing the final field at the CCD plane,

$$
E_{4}\left(\mathbf{r}_{4}\right)=\int E_{3}\left(\mathbf{r}_{3}\right) \exp \left[i P\left(\mathbf{r}_{3}\right)\right] \exp \left(\frac{2 \pi i}{\lambda f_{5}} \mathbf{r}_{3} \cdot \mathbf{r}_{4}\right) d \mathbf{r}_{3}
$$

The reference field $R\left(\mathbf{r}_{1}^{\prime}\right)$ is just a Gaussian beam codded in the hologram and it propagates through the same optical components. In order to allow an analytical calculation of the correlation function we suppose, without loss of generality, that the for this field the transformations phases are $Q\left(\mathbf{r}_{2}^{\prime}\right)=P\left(\mathbf{r}_{3}^{\prime}\right)=0$. Therefore, the correlation function is written $\mathrm{as}^{25}$,

$$
\begin{aligned}
\left\langle E_{4}^{*}\left(\mathbf{r}_{4}\right) E^{\prime}{ }_{4}\left(\mathbf{r}^{\prime}{ }_{4}\right)\right\rangle= & \int S^{*}\left(\mathbf{r}_{1}\right) R\left(\mathbf{r}_{1}^{\prime}\right)\left\langle E_{1}^{*}\left(\mathbf{r}_{1}\right) E_{1}\left(\mathbf{r}_{1}^{\prime}\right)\right\rangle \exp \left[-i Q\left(\mathbf{r}_{2}\right)\right] \exp \left(-\frac{2 \pi i}{\lambda f_{3}} \mathbf{r}_{2} \cdot \mathbf{r}_{3}\right) \\
& \times \exp \left[-i P\left(\mathbf{r}_{3}\right)\right] \exp \left(-\frac{2 \pi i}{\lambda f_{5}} \mathbf{r}_{3} \cdot \mathbf{r}_{4}\right) \exp \left(\frac{2 \pi i}{\lambda f_{3}} \mathbf{r}_{2}^{\prime} \cdot \mathbf{r}_{3}^{\prime}\right) \exp \left(\frac{2 \pi i}{\lambda f_{5}} \mathbf{r}_{3}^{\prime} \cdot \mathbf{r}_{4}^{\prime}\right) \\
& \times \exp \left(-\frac{2 \pi i}{\lambda f_{4}} \mathbf{r}_{1} \cdot \mathbf{r}_{2}\right) \exp \left(\frac{2 \pi i}{\lambda f_{4}} \mathbf{r}_{1}^{\prime} \cdot \mathbf{r}_{2}^{\prime}\right) d \mathbf{r}_{1} d \mathbf{r}_{2} d \mathbf{r}_{3} d \mathbf{r}^{\prime}{ }_{1} d \mathbf{r}_{2}^{\prime}{ }_{2} d \mathbf{r}^{\prime}{ }_{3},
\end{aligned}
$$

where the symbol "*” stands for the complex conjugated field and $\langle.$.$\rangle means an ensemble average.$

We consider that the speckle fields are delta-correlated, i.e., its cross-correlation at the plane of the hologram that generates the signal and reference fields is $\left\langle E_{1}^{*}\left(\mathbf{r}_{1}\right) E_{1}\left(\mathbf{r}_{1}^{\prime}\right)\right\rangle=\delta\left(\mathbf{r}_{1}-\mathbf{r}_{1}^{\prime}\right)$. Therefore, the integral in $\mathbf{r}_{1}$ and $\mathbf{r}_{1}^{\prime}$ can be evaluated resulting in

$$
\widetilde{S}^{*}\left(\mathbf{r}_{2}-\mathbf{r}_{2}^{\prime}\right) * \widetilde{R}\left(\mathbf{r}_{2}-\mathbf{r}_{2}^{\prime}\right)=\int S^{*}\left(\mathbf{r}_{1}\right) R\left(\mathbf{r}_{1}\right) \exp \left(-\frac{2 \pi i}{\lambda f_{4}} \mathbf{r}_{1} \cdot\left(\mathbf{r}_{2}-\mathbf{r}_{2}^{\prime}\right)\right) d \mathbf{r}_{1} .
$$

Equation (9) represents a convolution between the Fourier transformed $\operatorname{sign} \widetilde{S}^{*}\left(\mathbf{r}_{2}-\mathbf{r}_{2}^{\prime}\right)$ and reference fields $\widetilde{R}\left(\mathbf{r}_{2}-\mathbf{r}_{2}^{\prime}\right)$. Since the waist size of the reference beam is bigger than that of the signal beam, we can make the 

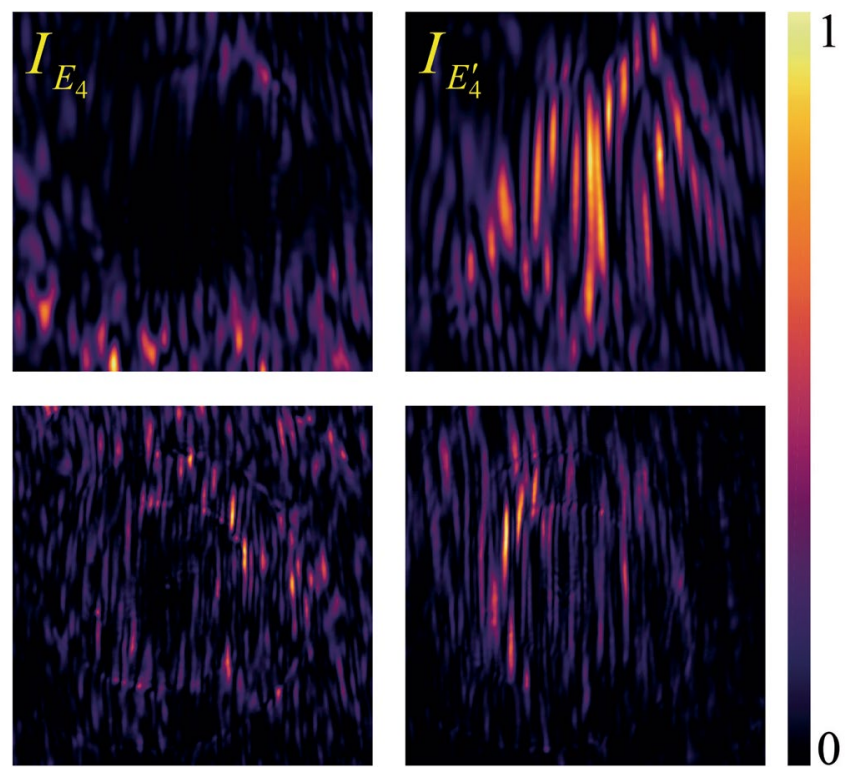

Figure 3. Random intensities patterns observed for the signal $I_{E_{4}}=\left|E_{4}\right|^{2}(m=2$, first column) and reference beams $I_{E^{\prime}}=\left|E^{\prime}{ }_{4}\right|^{2}(m=0$, second column) at the detection plane. First line simulation and second line experiment. The windows are squares of $1.4 \mathrm{~mm} \times 1.4 \mathrm{~mm}$.

approximation $\widetilde{R}\left(\mathbf{r}_{2}-\mathbf{r}_{2}^{\prime}\right) \approx \delta\left(\mathbf{r}_{2}-\mathbf{r}_{2}^{\prime}\right)$ implying that $\widetilde{S}^{*}\left(\mathbf{r}_{2}-\mathbf{r}_{2}^{\prime}\right) * \widetilde{R}\left(\mathbf{r}_{2}-\mathbf{r}_{2}^{\prime}\right) \approx \widetilde{S}^{*}\left(\mathbf{r}_{2}-\mathbf{r}_{2}^{\prime}\right)$. We also note that the integral in $\mathbf{r}_{3}^{\prime}$ in Eq. (8) results $\delta\left(f_{5} \mathbf{r}_{2}^{\prime}+f_{3} \mathbf{r}_{4}^{\prime}\right)$. Using all of these results Eq. (8) simplifies to,

$$
\left\langle E_{4}^{*}\left(\mathbf{r}_{4}\right) E^{\prime}{ }_{4}\left(\mathbf{r}^{\prime}{ }_{4}\right)\right\rangle=\int \widetilde{S}^{*}\left(\mathbf{r}_{2}+\mathbf{r}^{\prime}{ }_{4} \frac{f_{3}}{f_{5}}\right) \exp \left[-i Q\left(\mathbf{r}_{2}\right)-i P\left(\mathbf{r}_{3}\right)\right] \times \exp \left(-\frac{2 \pi i}{\lambda f_{4}} \mathbf{r}_{2} \cdot \mathbf{r}_{3}\right) \exp \left(-\frac{2 \pi i}{\lambda f_{4}} \mathbf{r}_{3} \cdot \mathbf{r}_{4}\right) d \mathbf{r}_{2} d \mathbf{r}_{3} .
$$

Using $\mathbf{r}_{4}=\mathbf{r}$ and $\mathbf{r}_{4}^{\prime}=0$ we realize that the correlation function $\left\langle E_{4}^{*}(\mathbf{r}) E^{\prime}{ }_{4}(0)\right\rangle$ is identical with the result that could be obtained for the coherent fields that arrives at the final plane at the $\mathrm{CCD}^{10}$. There is no analytical result for the integral in Eq. (10) therefore, in the next section, we present a numerical simulation which is equivalent to the effect described by this integral.

Numerical simulation and experimental results. For the numerical simulation we have used the band-limited angular spectrum method ${ }^{29}$ to propagate in the free space between the lenses and the SLMs, where each lens of focal length $f_{i}$ is represented by a quadratic phase $\exp \left[-i k r^{2} /\left(2 f_{i}\right)\right]$. Therefore, the field is multiplied by this quadratic phase to simulate the crossing of a lens. Similarly, the beam is multiplied by a phase $\exp [i Q(x, y)]$ or $\exp [i P(x, y)]$ to simulate the reflection of the field in the SLMs. We have first to generate a speckle field which is multiplied by the LG beams and then propagated though the lens of focus $f_{4}$ until the transformation phase $Q(x, y)$, and after that it follows the path through the lens of focus $f_{3}$ until the correction phase $P(x, y)$, and then through the lens $f_{5}$ until the CCD camera. To generate a speckle field we have multiplied a Gaussian function by a random phase and performed a Fourier transform of it. The size of the speckles where very small such that we can consider the field as a delta-correlated field. The reference beam is generated just passing a zero order LG beam through the system.

In the experiment, we measured the signal intensity followed by the reference intensity with RGGD stopped and then rotate the RGGD a small angle and stop it again to measure the next pair of signal and reference beams. For each pair of measurement we need to calculate the cross-correlation between the measured signal and reference intensities and we average over 100 measurements. Thanks to the Reed's momentum theorem ${ }^{30}$ the intensity cross-correlation $\Gamma$ can be written as ${ }^{31,32}$,

$$
\Gamma=A+\left|\left\langle E_{4}^{*} E_{4}^{\prime}\right\rangle\right|^{2}
$$

where $A$ is a background and $W=\left\langle E_{4}^{*} E^{\prime}{ }_{4}\right\rangle$ is given by Eq. 10 .

Figure 3 shows samples of the numerically calculated (first line) and experimentally measured speckle patters (second line), for the signal (first column) and reference beams (second column) at the CCD plane.

Figure 4, in the first and second columns, presents the results for the intensity cross-correlation patterns $\Gamma$ for the simulation and experiment, respectively. Although the signal and reference beams fluctuates randomly, the correlation function between them tends to a well-defined pattern in agreement with the prediction of the theory. We can observe that another prediction of the theory is also confirmed, i. e., despite of a background, the patterns for the cross-correlation $|W|^{2}$ was the same that the intensities of the coherent fields in ref. ${ }^{10}$. 


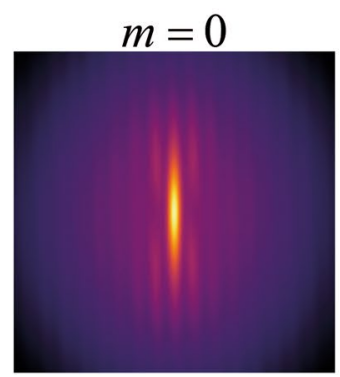

$m=1$

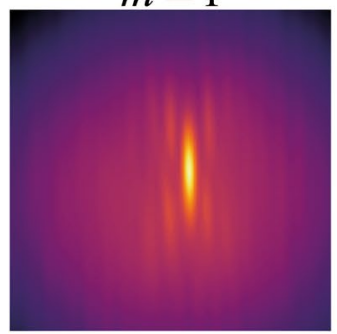

$m=-1$

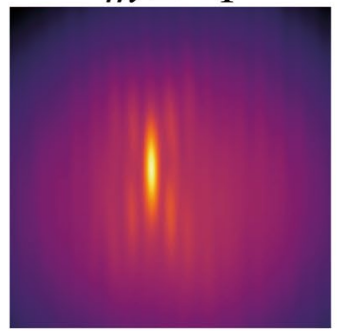

$m=2$

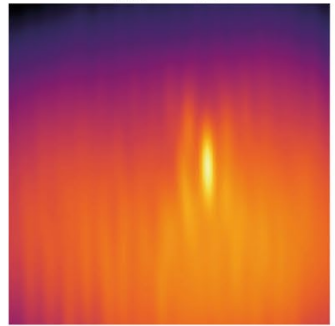

$m=-2$

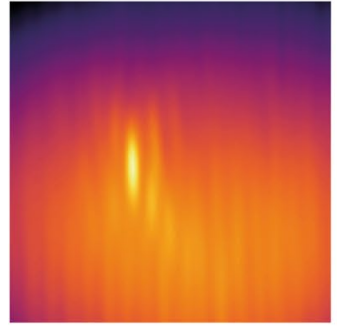

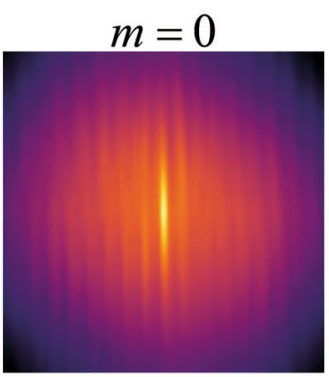

$m=1$

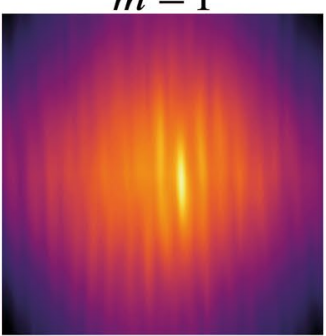

$m=-1$

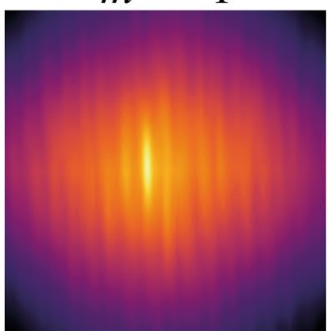

$m=2$

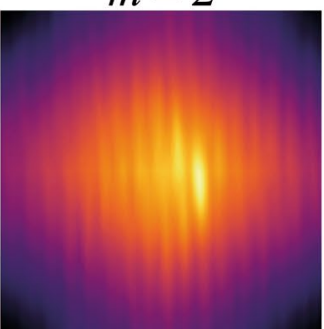

$m=-2$

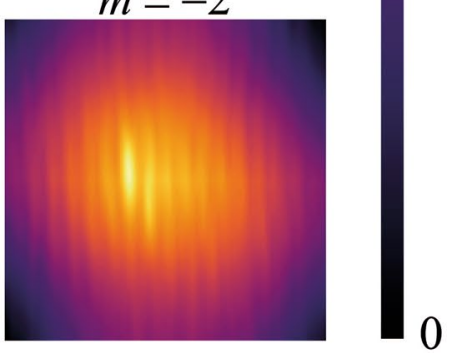

Figure 4. Simulation (first column) and experimental results (second column) for the intensity crosscorrelation functions $\Gamma$. The windows are squares of $1 \mathrm{~mm} \times 1 \mathrm{~mm}$.

Figure 5 shows transversal profiles taken at the center of the simulation (first column of Fig. 4) of the cross-correlation functions of the spatially incoherent field, and the same for the experimental results (second column of Fig. 4). In the experiment and simulation of the cross-correlation functions we have averaged over 100 calculations of the cross-correlation through 100 realization of the pair of random pattern (see Fig. 2) and have performed a background subtraction ${ }^{33}$, resulting $|W|^{2}$. The distance between two adjacent peaks is $\lambda f_{5} /(2 \pi \beta)=59.1 \mu \mathrm{m}^{10}$. The distance between adjacent peaks obtained directly by the image in the CCD camera is $59.5 \mu \mathrm{m}$, close to that value. Using this distance we can extract the topological charge from the distance of each mode to the central zero order mode. Therefore, the only previous calibration that we have to do is determining the position the zero order mode. We observe a good agreement between simulation and experiment indicating that the sorting scheme is viable with spatially incoherent light. 

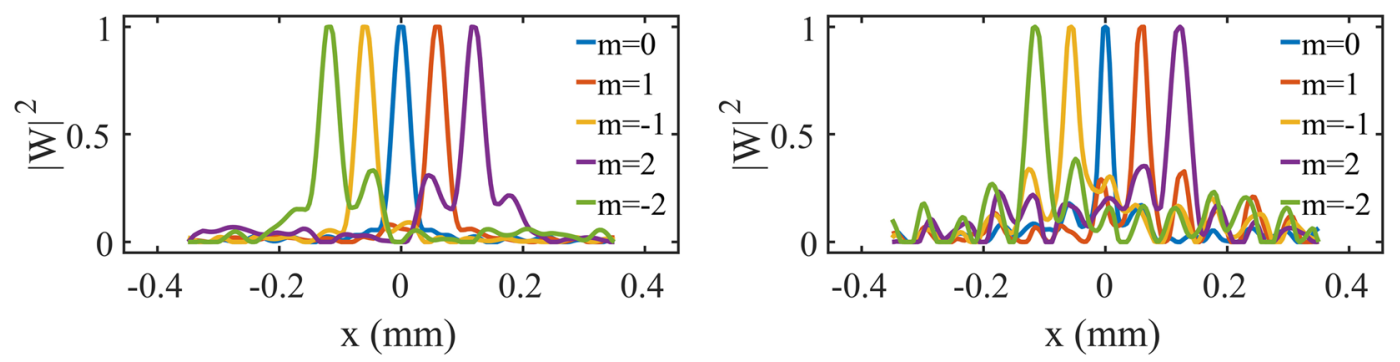

Figure 5. Profiles for the simulation (fist column) and experiment (second column) for the field crosscorrelation function $|W|^{2}$.

\section{Conclusion}

We have observed that it is possible the use the spatial degrees of freedom of the coherence function to spatially multiplex coherence vortex to encode information for optical communications. Besides we note that it is possible to implement the sorting of spatially multiplexed spatially incoherent vortex states. Considering that spatially incoherent optical vortices beams are robust against scattering by the atmospheric turbulence and opaque obstacles, it may constitute a promising alternative for codding and transmitting information.

\section{Methods}

Devices in the experimental setup. A have used a laser model Ultralasers MSL-FN-532-200mW operating at $532 \mathrm{~nm}$. The SLM ${ }_{1}$ used in the experiments is a Holoeye LETO and the SLM $\mathrm{S}_{2}$ is a Hamamatsu X10468-01, both are phase-only SLMs based on reflective LCOS microdisplays. The Holoeye LETO SLM have a spatial resolution of $1920 \times 1080$ pixels and a pixel size of $6.4 \mu \mathrm{m}$ and the Hamamatsu SLM have a spatial resolution of $600 \times 792$ pixels and a pixel size of $20 \mu \mathrm{m}$. The resolution of the phase patterns displayed on the Holoeye SLM are $960 \times 960$ pixels, square shape and displayed side by side at the center. The resolution of the phase pattern displayed on the Hamamatsu SLM is $600 \times 600$ pixels displayed at the center. The camera used in the experiment is a PixeLink PL-B781F.

Received: 23 August 2019; Accepted: 26 December 2019;

Published online: 13 February 2020

\section{References}

1. Allen, L., Barnett, S. M. \& Padgett, M. J. Optical angular momentum. (CRC press, (2016).

2. Wang, J. et al. Terabit free-space data transmission employing orbital angular momentum multiplexing. Nature photonics 6, 488 (2012).

3. Bozinovic, N. et al. Terabit-scale orbital angular momentum mode division multiplexing in fibers. science 340, 1545-1548 (2013).

4. Molina-Terriza, G., Torres, J. P. \& Torner, L. Management of the Angular Momentum of Light: Preparation of Photons in Multidimensional Vector States of Angular Momentum. Physical Review Letters 88, 013601, https://doi.org/10.1103/ PhysRevLett.88.013601 (2001).

5. García-Escartín, J. C. \& Chamorro-Posada, P. Quantum multiplexing with the orbital angular momentum of light. Physical Review A 78, 062320, https://doi.org/10.1103/PhysRevA.78.062320 (2008).

6. Allen, L., Beijersbergen, M. W., Spreeuw, R. J. \& Woerdman, J. P. Orbital angular momentum of light and the transformation of Laguerre-Gaussian laser modes. Phys Rev A 45, 8185-8189 (1992).

7. Willner, A. E. et al. Optical communications using orbital angular momentum beams. Adv. Opt. Photon. 7, 66-106, https://doi. org/10.1364/AOP.7.000066 (2015).

8. Berkhout, G. C., Lavery, M. P., Courtial, J., Beijersbergen, M. W. \& Padgett, M. J. Efficient sorting of orbital angular momentum states of light. Physical review letters 105, 153601 (2010).

9. Mirhosseini, M., Malik, M., Shi, Z. \& Boyd, R. W. Efficient separation of the orbital angular momentum eigenstates of light. Nature communications 4, 2781 (2013).

10. Wen, Y. et al. Spiral Transformation for High-Resolution and Efficient Sorting of Optical Vortex Modes. Physical Review Letters 120, 193904, https://doi.org/10.1103/PhysRevLett.120.193904 (2018).

11. Leach, J., Padgett, M. J., Barnett, S. M., Franke-Arnold, S. \& Courtial, J. Measuring the Orbital Angular Momentum of a Single Photon. Physical Review Letters 88, 257901, https://doi.org/10.1103/PhysRevLett.88.257901 (2002).

12. Zhang, W., Qi, Q., Zhou, J. \& Chen, L. Mimicking Faraday Rotation to Sort the Orbital Angular Momentum of Light. Physical Review Letters 112, 153601, https://doi.org/10.1103/PhysRevLett.112.153601 (2014).

13. Hossack, W. J., Darling, A. M. \& Dahdouh, A. Coordinate Transformations with Multiple Computer-generated Optical Elements. Journal of Modern Optics 34, 1235-1250, https://doi.org/10.1080/09500348714551121 (1987).

14. Palacios, D. M., Maleev, I. D., Marathay, A. S. \& Swartzlander, G. A. Spatial Correlation Singularity of a Vortex Field. Physical Review Letters 92, 143905, https://doi.org/10.1103/PhysRevLett.92.143905 (2004).

15. Salla, G. R., Perumangattu, C., Prabhakar, S., Anwar, A. \& Singh, R. P. Recovering the vorticity of a light beam after scattering. Applied Physics Letters 107, 021104, https://doi.org/10.1063/1.4926913 (2015).

16. Reddy, S. G., Kumar, A., Prabhakar, S. \& Singh, R. P. Experimental generation of ring-shaped beams with random sources. Opt. Lett. 38, 4441-4444, https://doi.org/10.1364/OL.38.004441 (2013).

17. Jesus-Silva, A. J., Hickmann, J. M. \& Fonseca, E. J. S. Strong correlations between incoherent vortices. Opt. Express 20, 19708-19713, https://doi.org/10.1364/OE.20.019708 (2012).

18. Alves, C. R., Jesus-Silva, A. J. \& Fonseca, E. J. S. Characterizing coherence vortices through geometry. Opt. Lett. 40, 2747-2750, https://doi.org/10.1364/OL.40.002747 (2015).

19. Ponomarenko, S. A. A class of partially coherent beams carrying optical vortices. J. Opt. Soc. Am. A 18, 150-156, https://doi. org/10.1364/JOSAA.18.000150 (2001). 
20. Wang, T., Pu, J. \& Chen, Z. Propagation of partially coherent vortex beams in a turbulent atmosphere. Optical Engineering 47(1-5), 5 (2008).

21. Alves, C. R., Jesus-Silva, A. J. \& Fonseca, E. J. S. Robustness of a coherence vortex. Appl. Opt. 55, 7544-7549, https://doi.org/10.1364/ AO.55.007544 (2016)

22. Chen, J. \& Li, Y. Discrimination of incoherent vortex states of light. Opt. Lett. 43, 5595-5598, https://doi.org/10.1364/OL.43.005595 (2018).

23. Krenn, M. et al. Communication with spatially modulated light through turbulent air across Vienna. New Journal of Physics 16, 113028, https://doi.org/10.1088/1367-2630/16/11/113028 (2014).

24. Goodman, J. W. Introduction to Fourier Optics. (W. H. Freeman, (2005).

25. Mandel, L. \& Wolf, E. Optical Coherence and Quantum Optics. (Cambridge University Press, (1995).

26. Cai, Y. \& Zhu, S.-Y. Ghost interference with partially coherent radiation. Opt. Lett. 29, 2716-2718, https://doi.org/10.1364/ OL.29.002716 (2004).

27. Vidal, I., Caetano, D. P., Fonseca, E. J. S. \& Hickmann, J. M. Effects of pseudothermal light source's transverse size and coherence width in ghost-interference experiments. Opt. Lett. 34, 1450-1452, https://doi.org/10.1364/OL.34.001450 (2009).

28. Arrizón, V., Ruiz, U., Carrada, R. \& González, L. A. Pixelated phase computer holograms for the accurate encoding of scalar complex fields. J. Opt. Soc. Am. A 24, 3500-3507, https://doi.org/10.1364/JOSAA.24.003500 (2007).

29. Matsushima, K. \& Shimobaba, T. Band-Limited Angular Spectrum Method for Numerical Simulation of Free-Space Propagation in Far and Near Fields. Opt. Express 17, 19662-19673, https://doi.org/10.1364/OE.17.019662 (2009).

30. Reed, I. On a moment theorem for complex Gaussian processes. IRE Transactions on Information Theory 8, 194-195, https://doi. org/10.1109/TIT.1962.1057719 (1962).

31. Rodrigues, J. S., Fonseca, E. J. S. \& Jesus-Silva, A. J. Talbot effect with partially coherent interfering Bessel beams. Appl. Opt. 57, 3186-3190, https://doi.org/10.1364/AO.57.003186 (2018).

32. Jesus-Silva, A. J., Silva, J. G., Monken, C. H. \& Fonseca, E. J. S. Experimental cancellation of aberrations in intensity correlation in classical optics. Physical Review A 97, 013832, https://doi.org/10.1103/PhysRevA.97.013832 (2018).

33. Cao, A. et al. A robust method for automated background subtraction of tissue fluorescence. Journal of Raman Spectroscopy 38 , 1199-1205, https://doi.org/10.1002/jrs.1753 (2007).

\section{Acknowledgements}

This work was supported by Coordenação de Aperfeiçoamento de Pessoal de Nível Superior (CAPES), Conselho Nacional de Desenvolvimento Científico e Tecnológico (CNPq), Fundação de Amparo à Pesquisa do Estado de Alagoas (FAPEAL), and Instituto Nacional de Ciência e Tecnologia de Informação Quântica (INCT-IQ).

\section{Author contributions}

Daniel O. Bezerra and João P. Amaral performed the experiment, Eduardo J. S. Fonseca, Cleberson R. Alves and Alcenísio J. Jesus-Silva conceived the experiment and wrote the paper.

\section{Competing interests}

The authors declare no competing interests.

\section{Additional information}

Correspondence and requests for materials should be addressed to A.J.J.-S.

Reprints and permissions information is available at www.nature.com/reprints.

Publisher's note Springer Nature remains neutral with regard to jurisdictional claims in published maps and institutional affiliations.

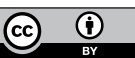

Open Access This article is licensed under a Creative Commons Attribution 4.0 International License, which permits use, sharing, adaptation, distribution and reproduction in any medium or format, as long as you give appropriate credit to the original author(s) and the source, provide a link to the Creative Commons license, and indicate if changes were made. The images or other third party material in this article are included in the article's Creative Commons license, unless indicated otherwise in a credit line to the material. If material is not included in the article's Creative Commons license and your intended use is not permitted by statutory regulation or exceeds the permitted use, you will need to obtain permission directly from the copyright holder. To view a copy of this license, visit http://creativecommons.org/licenses/by/4.0/.

(C) The Author(s) 2020 